\title{
The potential contribution of nanotechnology to nutritional well being
}

\author{
Jeremy J. Ramsden ${ }^{\dagger, *}$, \\ ${ }^{\dagger}$ Division of Biosystems, Tokyo Medical and Dental University (TMDU), 2-3-10 Kanda-Surugadai, Chiyoda-ku, \\ Tokyo 101-0062, Japan \\ *entre for Microsystems and Nanotechnology, Cranfield University, Bedfordshire, MK43 0AL, UK
}

\begin{abstract}
The history of food production, ranging from agriculture to industrial processing, is briefly surveyed. The potential contributions of nanotechnology, direct, indirect and conceptual, are assessed, both in the short and long terms. Both technological and political challenges are addressed.
\end{abstract}

Keywords: agriculture, food production, malnutrition, processing

\section{INTRODUCTION}

Hunters and gatherers - our ancestors until about 10000 years ago - presumably invariably ate fresh food. Given that degradation sets in within seconds of an animal being killed or a fruit or vegetable being plucked, this would appear to be a highly commendable state of affairs, conducive to good health. Nevertheless, hunting and gathering implies that most of man's waking hours had to be spent in search of food: without leisure, little development of civilization is possible.

The cultivation of plants, and the domestication of animals, allowed far greater control of food supply and was far more productive, ${ }^{1}$ enabling some time to be set aside for leisure, which itself provided opportunities to develop technology for dealing with the food surpluses that would have accrued at certain times of the year. A variety of familiar preservation technologies - such as butter and cheese, and salt beef and fish-would appear to be very ancient. Other technologies, associated with ploughs (drawn by animals) and irrigation, began to be developed, and other food processing technologies such as making wine and bread emerged (approximately 6000 and 5000 B.C. in Georgia and in Egypt respectively), and agrarian societies, based on large-scale farming, emerged about 5000 years ago. Since then, there has been a continuous expansion of this sector of activity, to the extent that at least in the developed world, probably a majority of food is processed after harvest. The introduction of cold storage by the Vestey brothers and others about a century ago ranks as a major milestone in this development. There is a common thread running through it, namely enhancing productivity allows surpluses to be accumulated, which both enable the leisure that can be used to develop new technology, and require such technology to prevent them from being wasted.

Since technology is a collective enterprise, it naturally fosters urbanization, ${ }^{2}$ which has been another notable trend ever since the emergence of agrarian societies. The greater the degree of urbanization, the less practicable it is to provide everyone with fresh food, hence processing for the purpose of preserving the comestibility of food becomes more and more necessary. In our present society, this has been extended to include the availability of exotic foods from almost every part of the world, which is nowadays expected as a matter of course even in a small town. ${ }^{3}$

Technology is thus inextricably intertwined with the provision of food, and has been for millennia. Now that we stand on the threshold of yet another technological revolution, this time the one associated with nanotechnology, it would appear to be unexceptionable to expect that it will also be exploited by the food industry.

\section{NANOTECHNOLOGY}

First, let us briefly recall what nanotechnology is. The bald definition is "the design, characterization, production and application of materials, devices and systems by controlling shape and size of the nanoscale" [1]. A slightly

\footnotetext{
*E-mail: j.ramsden@cranfield.ac.uk

1 In most places, that is to say. There are still regions of the world where soil fertility is very low.

2 No causal link is implied one way or the other here. If people started grouping their dwellings together for reasons other than the development of technology, such a society would clearly provide a good environment for such a development; on the other hand it seems to be equally plausible to imagine that the invention of a technology by an individual would tend to bring people together, not least because most technologies rely on other feeder ones for their practical realization. Furthermore, most technologies rely on a certain degree of division of labour to be effective, which is clearly only feasible in a reasonably large community.

3 This expectation has a complex origin. It is partly due to the availability of sufficient wealth to be able to afford exotic foods, and partly to the globalization of trade.
} 
different nuance is given by "the deliberate and controlled manipulation, precision placement, measurement, modelling and production of matter at the nanoscale in order to create materials, devices, and systems with fundamentally new properties and functions" [1]. The nanoscale itself is consensually considered to cover the range from 1 to $100 \mathrm{~nm}$. The emphasis on control is particularly important: it is this that distinguishes nanotechnology and chemistry, for example. A very succinct definition of nanotechnology is simply "engineering with atomic precision". Elaborating somewhat on the definitions, one can expand nanotechnology along at least three imaginary axes: the first one concerns tangible objects in order of increasing complexity: materials, devices and systems. Note that the boundaries between these three can be crossed by such things as "smart" materials. The second axis starts with static things such as materials and devices qua assemblages without explicitly considering dynamical aspects of their function, and proceeds through their manufacture (nanomanufacturing, usually abbreviated to nanofacture), i.e. processes, to nanometrology, which comprises a very varied collection of instruments and procedures. A third useful axis starts with direct nanotechnology: materials structured at the nanoscale (including nanoparticles), devices within the nanoscale, etc.; continues with indirect nanotechnology, which encompasses things like hugely powerful information processors based on very large scale integrated chips with individual circuit components within the nanoscale; and finishes with conceptual nanotechnology, which means the scrutiny of engineering and other processes at the nanoscale in order to understand them better [2].

\section{NANOTECHNOLOGY AND FOOD}

From this description, it is apparent that there are a great many possibilities for nanotechnology to be used in the food industry. Let us examine axis number three, for example. Conceptual nanotechnology is probably the least controversial, and would cover attempts to understand nutrition from the molecular viewpoint, not only confining attention to the elemental composition of micronutrients, but also their material state. There is also some interest in "molecular gastronomy", a term coined by Nicolas Kurti and Hervé This in 1992 signifying the application of scientific laboratory techniques to cooking, and since then enthusiastically taken up by a variety of chefs around the world, although it remains very much a niche activity. Indirect nanotechnology is dominated by powerful microprocessors enabling computation to be all pervasive. The farmer using a geographical information system to control robots in his fields is making good use of that. In the future, butchers may routinely employ tomography on carcasses to help determine the optimal dissection. The tomography itself requires heavy computations; nanotechnology-enabled processing power may become powerful enough to enable the optimal dissection to be automatically determined. Even cold storage systemsand indeed the logistics of the entire global distribution system-are controlled by microprocessors. Direct nanotechnology would cover the use of nanoscale sensors, in consequence cheap and unobtrusive enough to be ubiquitous, to monitor the state of food, including possible contamination with pesticides, or infectious agents acquired in the factory, or deficiencies arising through improper operations in a restaurant kitchen. Driven by a plethora of scandals leading to food poisoning, sometimes on quite a large scale, this is perceived to be a very welcome development by the general public, significantly offsetting some of the disadvantages of the modern agro-industrial complex. Benefits of a similar nature are already resulting from the use of nanocoatings for packaging materials, enhancing their desirable gas permeability characteristics, and sometimes incorporating an indicator function able to respond to (e.g.) the premature leakage of oxygen into a sealed anaerobic package [3].

The contentious aspect of nanotechnology concerns the possibility of including nanoscale additives in foodanother manifestation of direct nanotechnology. Additives to enhance the nutritional value of food are already widespread in the processed food industry (a very common example is the addition of vitamin $\mathrm{C}$ to fruit juices). The idea behind using nanotechnology is to enhance the functionality and hence effectiveness of these additives - for example, encapsulating the vitamin C in minute hollow spheres made from calcium carbonate, so that the vitamin does not oxidize and become nutritionally valueless while the juice is standing in the air waiting to be drunk, but will only be released in the acidic environment of the stomach. Inasmuch as these additives are already becoming more and more sophisticated, introducing nanotechnology seems to be merely a continuation of an existing trend. Since nanotechnology is typically associated with achieving higher added-value for a product, it is natural that it is of particular interest in the rather young field of "neutraceuticals"-foodstuffs deliberately enhanced with substances that would rank as pharmaceuticals. This development has in itself not been free of controversy - probably the best-known example is the addition of fluoride to drinking water.

The fundamental argument against this kind of thing is that our physiology is not adapted to such novelties, and may not be able to adapt before some harm is done. This constitutes the basic objection to ingesting 
genetically modified foods. It is quite difficult to find the right level at which to address the problem. Clearly DNA as such is not in general toxic-we are eating it all the time. On the other hand, certain sequences, e.g. those of a virus, may be harmful, at least under certain circumstances. The situation recalls the debates over the quality of drinking water in London in the middle of the 19th century-certain experts likened the inadvertent consumption of microörganisms in the water supply as being no more dangerous than eating fish. Given the state of knowledge at the time, it would perhaps have been difficult to adduce irrefutable arguments against that viewpoint. The only way to proceed is to build up knowledge that can be applied to calculate risks, and weighed against possible benefits. Provided the knowledge is there, this can be done quite objectively and reliably, ${ }^{4}$ but gaining the knowledge is likely to be a laborious task, especially when it comes to assessing the chronic effects resulting from many years of low-level exposure. There is particular anxiety regarding the addition of small metallic or metal oxide nanoparticles to food. Although a lot about their biological effects is indeed already known [5], the matter is complex enough for the ultimate fates of such particles in human bodies to be still rather poorly understood, and new types of nanoparticles are being made all the time. ${ }^{5}$ On the other hand, it is also worth bearing in mind that some kinds of nanoparticles have been around for a long time-volcanoes and forest fires generate vast quantities of dust and smoke, virus particles are generally within the nanorange, comestible biological fluids such as milk contain soft nanoparticles, and so forth, merely considering natural sources. Anthropogenic sources include combustion in many forms, ranging from candles, oil lamps, tallow dips etc. used for indoor lighting, internal combustion engines - this is a major source of nanoparticle pollution in cities, along with the dust generated from demolishing buildings - cooking operations, and recreational smoking. The occupational hazards from certain industries, especially mining and mineral processing (silicosis, asbestosis), are well recognized, and the physicochemical and immunological aspects on the hazards of the nanoparticles reasonably well understood [6].

A good example of how nanoscale knowledge has led to a profoundly new understanding of previously unsuspected hazards is provided by the discovery, using the black lipid membrane (BLM) technique [7], that certain cyclic polyunsaturated compounds synthesized by bacteria used in the biotechnological production of artificial sweeteners are able to form ion channels in our cell membranes. Trace quantities of these compounds remain in the so-called "high energy" and other soft drinks that seem to enjoy a growing popularity, and may be responsible for the growing incidence of heart problems among teenagers in societies where the consumption of these beverages has become the norm. It is another matter, however, to diffuse this knowledge among the general public, in order that they may weigh the risks against the supposed enjoyment.

The possibility of choice is an important aspect of the current debate on the matter. Ideally, if knowledge is insufficient for it to be clear whether benefits or risks are preponderant, a product should be available both with and without the nanoadditive. Then the consumer can make his or her choice-caveat emptor. ${ }^{6}$ In reality, it is well known that choice tends to contract. For example, nearly all the world's soybeans come from a certain (genetically modified) variety; $99 \%$ of tomatoes grown in Turkey are no longer indigenous varieties. ${ }^{7}$ It appears to be empirically well established that mysterious "market forces" drive matters to this result, and it needs to be taken into account when it is discussed whether nanoadditives should be permitted or not. We are familiar with the state of affairs in traditional (non-nano) in food processing. For example, it is possible to buy dairy products, such as cheese, made from either raw or pasteurized milk. For some consumers, avoiding the risk of contracting tuberculosis is the preponderant consideration; for others, the undesirability of consuming advanced glycation end products (AGEs, resulting from the chemical reaction between sugars and animal proteins or fats, typically occurring during frying or pasteurization) outweighs that risk; for yet others taste is the important consideration.

It is perhaps appropriate at this point to raise the question of regulation. Caveat emptor is a universal injunction, which should actually render regulation superfluous, since it is generally called for to protect the unsuspecting consumer from unscrupulous purveyors of goods or services, but if the consumer took the trouble to

\footnotetext{
4 Especially using the J-value [4].

5 It is actually quite inadequate to refer generically to nanoparticles. It is already known that their toxicity depends on size, shape and chemical constitution, and very possibly on their state of crystallinity (think of the problems of polymorphism of active ingredients in the pharmaceutical industry!). Therefore, at the very least some information on these characteristics should be provided when referring to "nanoparticles".

6 This, incidentally, highlights the importance of the members of society being sufficiently knowledgeable to be able to make an informed choice.

7 See [8] for further examples.
} 
properly investigate what he was letting himself in for, presumably the good or service would not be bought, and the unscrupulous purveyor would be less likely to continue to attempt to sell whatever it was, profit being the sole motive.

Nevertheless, despite repeatedly hearing that developed countries are "knowledge-based economies", it seems that the knowledge necessary to properly apply the principle of caveat emptor is lacking among the general public. Moreover, in many countries it is lacking among members of the legislative bodies. Therefore, one of the most urgent political needs is to ensure that parliamentarians and the like raise their level of knowledge and understanding of our technologically advanced society to ensure that they can effectively fill the knowledge gap between the technologists and the still largely ignorant consumer. A discussion of how this might be done goes beyond the scope of this article, however ${ }^{8}$

Less controversial than nanoadditives, but just as nano, are developments to achieve not a chemical modification of a foodstuff, but a modification to its structure. This particularly affects not taste, which is perhaps above all dependent on the actual chemicals sensed by our tastebuds, but mouthfeel, which is a very difficult characteristic of a natural foodstuff to imitate. Hence the food processing industry is devoting a great deal of ingenuity to find (with the application of nanometrology, since it does seem that physical structure at the nanoscale is responsible) ways of mimicking desirable mouthfeel, in products such as "ice cream" (which, if industrially manufactured, may contain very little cream).

\section{THE SOCIAL CONTEXT}

"Man ist, was man isst", as Martin Luther famously remarked in one of his Tischgespräche. Given the centrality of food in human existence, it can hardly be discussed in isolation as a purely physiological matter. Indeed, there is even evidence that the intake of folic acid by a pregnant mother can influence the methylation of the baby's proteins [9]. ${ }^{9}$ Furthermore, it is too much to expect that we always eat "sensible" foods, or carefully examine the list of ingredients on a packet (which anyway is usually woefully inadequate, particularly regarding the actual quantities of the substances mentioned), or acquire a personal biosensor for verifying the absence of hormone-active substances in vegetables sold on the market on the spot. It may be nowadays trite to repeat John Donne's dictum "No man is an island", but if anything it is even more true today, in a worldwide webconnected age, than it was at the end of the Middle Ages. We are all affected by the foods around us, whether we partake of them or not.

The dominant social aspect of nutrition is malnutrition coupled with obesity. It is estimated that current world production of food is adequate for the current world population, but much of that food is in the wrong place at the wrong time. Technologies, such as cold storage, and nanoparticle-containing gas-resistant wrappers, should therefore contribute to alleviating unevenness is of supply. The technologies come at a price, howeversome of them are expensive, either directly or indirectly - many modern farming practices, including intensive agriculture of all kinds, and fish farming, tend to yield produce that is less wholesome than their nonintensive counterparts.

The solution to eating the wrong foods - such as those that leave one undernourished, or overweight or both - is surely more knowledge. This is the perennial problem of a society based on high technology - it can only be truly successful if all members are sufficiently knowledgeable to properly partake in its development. Hence to properly cover the title theme of this article, we may also need to inquire how nanotechnology can contribute to the education of the population.

In the Introduction, it was suggested that improving the texture of processing food, so that it felt better in the mouth for example, was a rather neutral aspect of nanotechnology compared with the addition of physiologically active substances. But even the physical texture of a food has a social aspect. It is not only a matter of promoting sales of products in order to increase the revenues of the manufacturer: for example, processed food manufacturers are (commendably) trying to reduce the content of fat and sugar in their products (in order to reduce obesity), but even if they successfully mimic the taste of the original food, mouthfeel is difficult to imitate under these constraints, as already pointed out, and may result in a product being commercially unsuccessful, however beneficial it might be for health, and hence unviable to manufacture further.

\footnotetext{
${ }^{8}$ Nevertheless, it is worth remarking that we seem to be no closer to the dilemma, repeatedly pointed out by dispassionate observers during the last 50 years, posed by two equally unsatisfactory possible solutions, namely: giving scientists control of our society; and allowing governments to be only very feebly influenced by rationality. New thinking to solve this problem is very much needed!

9 The methylation pattern of the repertoire of genes is a key controlling factor in development.
} 


\section{NANOTECHNOLOGYAND THE FOOD CRISIS}

At the beginning of this month (June) the Food and Agriculture Organization (FAO, part of the United Nations) held a three-day summit conference in Rome in order to explore ways of overcoming difficulties due to steeply rising food prices around the world, which have caused especially grave problems in poor countries. Although part of the problems lies in the commercial sphere, and may be dealt with by considering the effects of export restrictions and price controls, a sustainable solution clearly lies in the technological realm. In the short-term, charitable deliveries of seeds and fertilizers may alleviate the problem; in the medium term such measures are likely to make things worse. Therefore, a thorough appraisal of the state of agronomy is needed. In fact, a number of recent reports have pointed to the research deficit in the field during the last few decades [10]. Yet in its call for increasing public support (in the developing countries) for agronomy research, the FAO is essentially still thinking of traditional approaches. In view of the generally revolutionary nature of nanotechnology, it must be expected that here too, it can make a decisive contribution.

There seem to be two timescales involved. One covers the next few years, and is based on the intense nanoscale scrutiny of the processes of comestible biomass production in its entirety, followed by inspired intervention at that scale. An example is biological nitrogen fixation. A wealth of detail is already known about the process, at the molecular level (the nitrogenase enzymes responsible for actually fixing the nitrogen), at the microbiological level (the symbiotic rhizobia), and at the ecological level (soil and inoculation, although here there are still inexplicable mysteries). Intervention, e.g. with functionalized nanoparticles, especially to improve fixation in difficult (e.g., dry or saline) conditions, seems to be feasible. The actual need is for laboratory and field research to establish the possibilities and limitations of such an approach.

The more distant timescale involves the introduction of molecular manufacturing [11]. Should this ever come about, anything, including any kind of foodstuff, will be able to be made from acetylene and electrical power [12]. This, more than anything else, will revolutionize the world order; whether hunger is abolished will depend on politics (and demography).

\section{CONCLUSIONS}

Looking back over the past millennia of human civilization, improvements in technology have enormously increased agricultural output, but this has also led to a concomitant increase in world population, hence global nutritional difficulties remain. Geographical mismatch of supply and demand is also frequently mentioned as a contributor to malnutrition - somewhat ironically, in an age of unprecedentedly large global trade volumes. A serious current problem is that it is becoming increasingly clear that productivity increases tend to be quantitative and imply product quality decreases. This goes well beyond mere unpalatability. ${ }^{10}$ The output of the agroindustrial complex, including, or perhaps especially, residues of pesticides and the presence of hormoneactive substances, may solve the basic malnutrition problem, but may introduce new problems of ill health, which may be deeply unsustainable, although the manufacturers of pharmaceuticals may see it as a source of new opportunities.

\section{ACKNOWLEDGMENTS}

The author would like to record his sincere thanks to Kenji Yasuda for his invitation to serve as visiting professor at TMDU, during the tenure of which this article was drafted out. He also thanks Pavel A. Grigoriev of the Institute of Cell Biophysics, Pushchino for having called his attention to the presence of ionophores in soft drinks containing artificial sweeteners, and Mohammad Ghalamboran for many interesting discussions about the possible application of nanotechnology to the problem of biological nitrogen fixation.

\section{REFERENCES}

1. Abad, E. et al. NanoDictionary. Basel: Collegium Basilea (2005); Terminology For the Bio-Nano Interface (PAS 132). London: British Standards Institute (2007).

2. Ramsden, J.J. What is nanotechnology? Nanotechnol. Perceptions 1 (2005) 3-17.

3. Ramsden, J.J. Nanotechnology in Coatings, Inks and Adhesives. Leatherhead: Pira International (2004).

4. Thomas, P.J., Stupples, M.A. and Alghaffar, M.A. The extent of regulatory consensus on health and safety expenditure. Part 1: Development of the J-value technique and evaluation of regulators' recommendations. Trans. IchemE Part B 84 (2006) 329-336.

5. Revell, P.A., The biological effects of nanoparticles. Nanotechnol. Perceptions 2 (2006) 283-298.

6. van Oss, C.J., Naim, J.O., Costanzo, P.M., Giese, R.F., Wu, W. and Sorling, A.F. Impact of different asbestos species and other mineral particles on pulmonary pathogenesis. Clays Clay Minerals 47 (1999) 697-707.

\footnotetext{
10 "Mere" perhaps belies the significant contribution of the enjoyment of food to social harmony, creativity etc.
} 
7. Grigoriev, P.A. Unified carrier-channel model of ion transfer across lipid bilayer membranes. J. Biol. Phys. Chem. 2 (2002) 77-79.

8. Ramsden, J.J. Complex technology: a promoter of security and insecurity. In: Complexity and Security (eds J.J. Ramsden and P.J. Kervalishvili), pp. 249-264. Amsterdam: IOS Press (2008).

9. McKay, J.A., Williams, E.A. and Mathers, J.C. Folate and DNA methylation during in utero development and aging.
Biochem. Soc. Trans 32 (2004) 1006-1007.

10. World Development Report 2008: Agriculture for Development. Washington (D.C.): World Bank (2007).

11. Drexler, K.E. Molecular engineering: an approach to the development of general capabilities for molecular manipulation. Proc. Natl Acad. Sci. USA 78 (1981) 5275-5278.

12. Freitas, R.A., Jr. Economic impact of the personal nanofactory. Nanotechnol. Perceptions 2 (2006) 111-126. 